\title{
The Effect of Chemical Information on the Spatial Distribution of Fruit Flies: II Parameterization, Calibration, and Sensitivity
}

\author{
Maarten de Gee ${ }^{\mathrm{a}}$, Marjolein E. Lof ${ }^{\mathrm{a}, \mathrm{b}, *}$, Lia Hemerik ${ }^{\mathrm{a}}$ \\ ${ }^{a}$ Biometris, Department of Mathematical and Statistical Methods, Wageningen University, \\ P.O. Box 100, 6700 AC Wageningen, The Netherlands \\ ${ }^{b}$ Laboratory of Entomology, Wageningen University, P.O. Box 8031, 6700 EH Wageningen, \\ The Netherlands
}

Received: 1 February 2007 / Accepted: 2 April 2008 / Published online: 9 September 2008 (c) The Author(s) 2008. This article is published with open access at Springerlink.com

\begin{abstract}
In a companion paper (Lof et al., in Bull. Math. Biol., 2008), we describe a spatio-temporal model for insect behavior. This model includes chemical information for finding resources and conspecifics. As a model species, we used Drosophila melanogaster, because its behavior is documented comparatively well.

We divide a population of Drosophila into three states: moving, searching, and settled. Our model describes the number of flies in each state, together with the concentrations of food odor and aggregation pheromone, in time and in two spatial dimensions. Thus, the model consists of 5 spatio-temporal dependent variables, together with their constituting relations. Although we tried to use the simplest submodels for the separate variables, the parameterization of the spatial model turned out to be quite difficult, even for this wellstudied species.

In the first part of this paper, we discuss the relevant results from the literature, and their possible implications for the parameterization of our model. Here, we focus on three essential aspects of modeling insect behavior. First, there is the fundamental discrepancy between the (lumped) measured behavioral properties (i.e., fruit fly displacements) and the (detailed) properties of the underlying mechanisms (i.e., dispersivity, sensory perception, and state transition) that are adopted as explanation. Detailed quantitative studies on insect behavior when reacting to infochemicals are scarce. Some information on dispersal can be used, but quantitative data on the transition between the three states could not be found. Second, a dose-response relation as used in human perception research is not available for the response of the insects to infochemicals; the behavioral response relations are known mostly in a qualitative manner, and the quantitative information that is available does not depend on infochemical concentration. We show how a commonly used Michaelis-Menten type dose-response relation (incorporating a saturation effect) can
\end{abstract}

\footnotetext{
*Corresponding author.

E-mail address: Marjolein.Lof@wur.nl (Marjolein E. Lof).
} 
be adapted to the use of two different but interrelated stimuli (food odors and aggregation pheromone). Although we use all available information for its parameterization, this model is still overparameterized. Third, the spatio-temporal dispersion of infochemicals is hard to model: Modeling turbulent dispersal on a length scale of $10 \mathrm{~m}$ is notoriously difficult. Moreover, we have to reduce this inherently three-dimensional physical process to two dimensions in order to fit in the two-dimensional model for the insects. We investigate the consequences of this dimension reduction, and we demonstrate that it seriously affects the parameterization of the model for the infochemicals.

In the second part of this paper, we present the results of a sensitivity analysis. This sensitivity analysis can be used in two manners: firstly, it tells us how general the simulation results are if variations in the parameters are allowed, and secondly, we can use it to infer which parameters need more precise quantification than is available now. It turns out that the short term outcome of our model is most sensitive to the food odor production rate and the fruit fly dispersivity. For the other parameters, the model is quite robust.

The dependence of the model outcome with respect to the qualitative model choices cannot be investigated with a parameter sensitivity analysis. We conclude by suggesting some experimental setups that may contribute to answering this question.

Keywords Parameterization $\cdot$ Sensitivity analysis $\cdot$ Chemotaxis $\cdot$ Spatial population dynamics $\cdot$ Integro-difference equations

\section{Introduction}

Animal aggregation is a common phenomenon in ecological systems (Parrish and Edelstein-Keshet, 1999; Wertheim et al., 2005). As the individuals can move freely, there must be a clue that guides them to the aggregate, so the individuals must pick up information and respond to it. The sensory cues involved herein can be visual, auditory, tactile, or chemical. In insects, chemical information conveyance is important. Insects trying to find a substrate or host plant use chemical information for long range detection; at smaller distances, sight also becomes important. The infochemicals to which a species reacts can be either substances emitted by its environment, such as food odors and plant volatiles, or substances that the species itself emits, such as aggregation pheromone.

In order to study the implications of infochemical use on the dynamics of a single species within a spatial context, we developed a model (Lof et al., 2008) that incorporates odor dispersion and the responses of organisms, taking Drosophila melanogaster as a model organism. As this species has been used as research model in many studies before, its behavior is documented comparatively well.

In our model, the drosophilid population is divided into three states, according to their activity: a searching state $S$ (with fly density $P_{S}$ ), in which individuals use infochemicals to find a suitable resource, a settled state $R$ (with fly density $P_{R}$ ), in which individuals spend a period of time on a resource, and a moving state $M$ (with fly density $P_{M}$ ), in which individuals actively move away from the resource. Hence, we have three state variables and three transitions. As the fruit flies use only the lowest layer (say, up to $2 \mathrm{~m}$ above ground level), we can model their density as functions that depend on time and two spatial variables. It should be noted that it would be easy to extend the model to three spatial 
variables; however, this seriously affects the run times for simulations with the model, and thereby its practical usefulness.

Relevant modeling questions for transitions between states are: (1) In the transition from the searching state $S$ to the settled state $R$, what is the relative importance of smell and vision on the fruit fly behavior, and how does this relation vary with the distance to the resource? (2) In the transition from $R$ to the moving state $M$, how long do fruit flies remain settled on their resource? and (3) In the transition from $M$ to the searching state $S$, how long (or how far) do fruit flies fly away from their resource before they start to search for a new resource?

There are also questions about the behavior of flies in a particular state that require an answer: (4) In the searching state $S$, how do individuals of Drosophila melanogaster search? Can they sample the infochemicals so frequently that they can smoothly follow a concentration gradient, or is the sampling rate so low that their flight pattern consists of connected line segments? And what is their sensory dose-response relation? (5) In the settled state $R$, the actual population dynamics take place. Here, an appropriate model must be chosen, possibly including scramble competition and/or an Allee effect. (6) In the moving state $M$, the fruit fly first actively covers some distance from its former resource; but how fast and how far?

There also are the physical laws for the spreading of the infochemicals: (7) How fast are the infochemicals produced, how fast do they evaporate, and how fast do the evaporated infochemicals disperse through the air? Furthermore, there is a difference in the vertical domain for the fruit flies and the infochemicals: whereas the fruit flies use only the lowest layer, a fraction of the infochemicals may disperse higher than this. Therefore (8), we have to estimate at what rate the infochemicals get out of reach.

Although we adopted quite basic models to describe these separate processes, there still are a large number of parameters in the resulting model. In the first part of this paper, we discuss how we use data that we found in the existing literature for the parameterization of the model. Despite all performed research, many parameterization questions remain unanswered: the experimental research was simply not aimed at mathematical model building at the level of detail that we aim at. Nevertheless, even with a model in which not all parameters are known, simulations can be done that lead to important ecological results and questions. This is what we set out to do in our companion paper (Lof et al., 2008), where we used the simulations to investigate the possible consequences of infochemicals on the spatial population dynamics. In the absence of a proper parameterization, the inferences are rather qualitative. Therefore, we also performed a sensitivity analysis on our parameter values, which we present in the fourth section of this paper; the results of this analysis can be used to determine the generality of our conclusions also for different parameter values.

\section{Parameterization of the model: short term dynamics}

Our model deals with Drosophila fruit flies that have to search for resources (i.e., yeastinfected apples), where they can eat, mate, and where females can oviposit. Details of the spatial model structure are given in the companion paper (Lof et al., 2008). In this model, the spatial domain is discretized into patches that can contain a resource item. The short term dynamics of the fruit flies is modeled with Eqs. (1)-(8), describing the redistribution 
of adult Drosophila population $P$ over three different states: the searching state (with density of flies $P_{S}$ ), the settled state (with density of flies $P_{R}$ ), and the moving state (with density of flies $P_{M}$ ).

$$
\begin{aligned}
R(x, y) & = \begin{cases}0 & \text { if there is no apple at position }(x, y) \text { at time } t \\
1 & \text { if there is an apple at position }(x, y) \text { at time } t\end{cases} \\
\frac{\partial}{\partial t} P_{S} & =D_{P} \nabla^{2} P_{S}-\nabla \cdot\left[v P_{S} \nabla f(F, A)\right]+\alpha_{2} P_{M}-\lambda R P_{S}, \\
\frac{\partial}{\partial t} P_{R} & =\lambda R P_{S}-\alpha_{1} P_{R} \\
\frac{\partial}{\partial t} P_{M} & =\alpha_{1} P_{R}-\alpha_{2} P_{M}, \\
f(F, A) & =\frac{F}{F_{0}+F}+\eta \frac{F A}{F_{0} A_{0}+F A} \\
\frac{\partial}{\partial t} F & =D_{I} \nabla^{2} F-\mu F+\theta_{F} R \\
\frac{\partial}{\partial t} A & =D_{I} \nabla^{2} A-\mu A+\omega A_{R}, \\
\frac{\partial}{\partial t} A_{R} & =\theta_{A} P_{R}-\omega A_{R} .
\end{aligned}
$$

Here $F$ and $A$ are the concentrations in the air of food odors and of aggregation pheromone, respectively. The amount of aggregation pheromone (in liquid or adsorbed form) at the resource is $A_{R}$, and $f$ is a sensory index function with a nonlinear interdependence on both infochemicals, to be explained in Section 2.3. The parameters in these model equations are given in Table 1 .

In Eqs. (2)-(8), we did not mention the time and spatial coordinates explicitly, but all dynamic variables depend on time and position: the fly densities $P_{S}, P_{R}$ and $P_{M}$ as well as the infochemical concentrations $F, A$ and the amount of liquid pheromone $A_{R}$. Clearly, the settled flies $\left(P_{R}\right)$ can only be present on the resource items. The fruit flies come into the moving state by actively flying away from their resources, but in Eq. (4), it is not stated explicitly how this movement is modeled. In our companion paper (Lof et al., 2008), we assume that the fruit flies actively fly away with a velocity $\rho$ from the resource in arbitrary directions. This process cannot be modeled with ordinary or partial differential equations; in an integro-difference equation it is modeled with a ring random dispersal kernel.

\subsection{Fruit fly behavioral parameters}

\subsubsection{Transition rates}

No quantitative information is available on the probability that a fruit fly actually settles on a resource after having detected it. Experimental work by Wertheim et al. (2002) showed that the numbers of fruit flies that settled quickly built up in the first 15 minutes of the experiment, subsequently followed by a more gradual increase up to on average 100 out of the 200-400 released fruit flies. However, it is hard to derive an estimate for a settling 
Table 1 The model parameters involved in the short time dynamics and their values as discussed in the text

\begin{tabular}{|c|c|c|c|}
\hline Name & Description & Value & Units \\
\hline$D_{P}$ & $\begin{array}{l}\text { Dispersal coefficient of at random } \\
\text { moving fruit flies }\end{array}$ & 0.058 & $\mathrm{~m}^{2} \min ^{-1}$ \\
\hline$\alpha_{1}$ & $\begin{array}{l}\text { Resource leaving rate of settled fruit } \\
\text { flies }\end{array}$ & 0.002 & $\min ^{-1}$ \\
\hline$\alpha_{2}$ & $\begin{array}{l}\text { Start-to-search rate of moving fruit } \\
\text { flies }\end{array}$ & 0.5 & $\min ^{-1}$ \\
\hline$\lambda$ & Settlement rate of searching fruit flies & 0.25 & $\min ^{-1}$ \\
\hline$\rho$ & $\begin{array}{l}\text { Velocity of movement away from the } \\
\text { resource }\end{array}$ & 1 & $\mathrm{~m} \mathrm{~min}^{-1}$ \\
\hline$F_{0}$ & Saturation parameter for food odors & 10 & $n g m^{-2}$ \\
\hline$A_{0}$ & $\begin{array}{l}\text { Saturation parameter for aggregation } \\
\text { pheromone }\end{array}$ & 0.04 & $n g \mathrm{~m}^{-2}$ \\
\hline$D_{I}$ & $\begin{array}{l}\text { Dispersion coefficient of } \\
\text { infochemicals }\end{array}$ & 1 & $\mathrm{~m}^{2} \min ^{-1}$ \\
\hline$\mu(720)$ & $\begin{array}{l}\text { Loss rate of infochemicals in a } \\
12 \text { hours period (measured from the } \\
\text { moment of production) }\end{array}$ & 0.025 & $\min ^{-1}$ \\
\hline$\mu(5)$ & $\begin{array}{l}\text { Loss rate of infochemicals in a } \\
5 \text { minutes period (measured from the } \\
\text { moment of production) }\end{array}$ & 0.171 & $\min ^{-1}$ \\
\hline$\theta_{F}$ & Food odor production by the resource & 2 & ng apple ${ }^{-1} \min ^{-1}$ \\
\hline$\theta_{A}$ & $\begin{array}{l}\text { Aggregation pheromone production } \\
\text { by settled fruit flies }\end{array}$ & 0.83 & ng fly ${ }^{-1} \min ^{-1}$ \\
\hline$\omega$ & $\begin{array}{l}\text { Evaporation rate of liquid aggregation } \\
\text { pheromone }\end{array}$ & $4.10^{-4}$ & $\min ^{-1}$ \\
\hline$v$ & Attraction to infochemicals & $5 D_{P}$ & $\mathrm{~m}^{2} \min ^{-1}$ \\
\hline$\eta$ & $\begin{array}{l}\text { Attraction ratio of food odor together } \\
\text { with aggregation pheromones relative } \\
\text { to the attraction to food odor alone }\end{array}$ & 2.51 & - \\
\hline
\end{tabular}

rate from their data, because the total number of flies is not exactly known. Nevertheless, the experiment suggests that there is a rather high probability that a fruit fly settles the moment it finds a resource, and we use $\lambda=0.25 \mathrm{~min}^{-1}$. With this value, $71 \%$ of the searching population that found a resource settles within an interval of 5 minutes (this is the time step size in our simulations).

The gradual increase after the quick settlement indicates that fruit flies remained on the resource for a period of time (Wertheim et al., 2002). Again, we adopt the simplest possible model: the probability of a settled fly leaving the resource per unit of time is constant, not depending on its residence time on that resource or on the local fruit fly density. This proportionality constant must be small as compared to $\lambda$ : we take $\alpha_{1}=$ $0.002 \mathrm{~min}^{-1}$. Then in each interval of 5 minutes, $99 \%$ of the settled population remains on the resource. 
We further assume that after leaving the resource, fruit flies quickly start searching for a new resource. We choose $\alpha_{2}=0.5 \mathrm{~min}^{-1}$. This means that on average a fruit fly moves away from a resource for 2 minutes, after which it starts to search for a new resource.

Using these parameter values, the fractions of $\underline{P}_{S}, \underline{P}_{R}$, and $\underline{P}_{M}$ in equilibrium (in a homogeneous domain) can be calculated. In equilibrium, the time derivatives in Eqs. (2)(4) vanish. In that case,

$$
\alpha_{2} \underline{P}_{M}=\alpha_{1} \underline{P}_{R}=\lambda \underline{P}_{S} .
$$

Using the fact that these fractions add up to 1, we obtain

$$
\begin{gathered}
\underline{P}_{M}=\frac{\alpha_{1} \lambda}{\alpha_{1} \alpha_{2}+\alpha_{1} \lambda+\alpha_{2} \lambda}, \\
\underline{P}_{R}=\frac{\alpha_{2} \lambda}{\alpha_{1} \alpha_{2}+\alpha_{1} \lambda+\alpha_{2} \lambda}, \\
\underline{P}_{S}=\frac{\alpha_{1} \alpha_{2}}{\alpha_{1} \alpha_{2}+\alpha_{1} \lambda+\alpha_{2} \lambda} .
\end{gathered}
$$

Hence, for $\lambda=0.25, \alpha_{1}=0.002$, and $\alpha_{2}=0.5$, we find that $\underline{P}_{M}=0.004, \underline{P}_{R}=0.988$, and $\underline{P}_{S}=0.008$. Thus, in a homogeneous world of suitable substrate, fruit flies would spend $98.8 \%$ of their time on the substrates and only $1.2 \%$ flying.

As these values hold only for a homogeneous domain, they are not very indicative for the actual proportions. Indeed, when the soil is covered with yeast-infected apples, the necessity for searching is virtually eliminated. In the real world, the resources are scarce and ephemeral; therefore, the above-mentioned percentages are rather unrealistic. As the flies must spend more time searching, the actual value for $\underline{P}_{S}$, is much higher (depending on the resource density), and the values for $\underline{P}_{R}$ and $\underline{P}_{M}$ are correspondingly lower. If the spatial layout is specified, one may compute the equilibrium fractions by solving the corresponding time-invariant partial differential equations. Based on a few of these experiments, we estimate that fruit flies spend $70 \%$ of their time on the resources and $30 \%$ dispersing.

\subsubsection{Fruit fly dispersal}

Usable data on the mobility of fruit flies are scarce. From the results reported by Timofeeff-Ressovsky and Timofeeff-Ressovsky (1941), we infer that Drosophila melanogaster moves $10 \mathrm{~m}$ or less per day in the field. As the mean dispersal distance of a twodimensional random dispersal process is $\sqrt{\pi D_{P} t}$, this would indicate a dispersal coefficient $D_{P}=30 \mathrm{~m}^{2}$ per day if this displacement could be attributed to a completely random movement of the entire fruit fly population. However, in our model a considerable part of the movement is attributed to active searching, and this reduces the dispersion coefficient. Therefore, a dispersion coefficient $D_{P}=12.5 \mathrm{~m}^{2}$ per day for the total population, as adopted by Etienne et al. (2002) seems to be realistic.

The dispersion coefficient $D_{P}=12.5 \mathrm{~m}^{2}$ per day relates to the displacement per day for the total population. Since we divided the population into two active states and one static state, the two active states also have to make up for the part of the population that does not disperse. When we estimate that fruit flies spend $70 \%$ of their time on the resources and $30 \%$ dispersing, the value $D=12.5 \mathrm{~m}^{2}$ per day for the total population gives 
$D_{P}=12.5 / 0.3=41.7 \mathrm{~m}^{2}$ per day for the searching population. With a day length of 12 hours, this is equivalent to $D_{P}=0.058 \mathrm{~m}^{2} \mathrm{~min}^{-1}$ (Table 1).

\subsubsection{Leaving the resource}

No information is available on the distance covered by fruit flies between leaving a resource and starting to search actively for a new one. It is obvious that they first have to move actively away from the resource, because a fruit fly that starts searching for a resource as soon as it leaves a resource only has a small probability of not returning to the same resource. We arbitrarily chose a flight speed of $\rho=1 \mathrm{~m} \mathrm{~min}^{-1}$, in a random direction from its leaving point, and a transition rate to the searching state $\alpha_{2}=0.5 \mathrm{~min}^{-1}$. This implies that the flies fly away from the resource during on average $2 \mathrm{~min}$ before they start to search again. During this time, they cover an average distance of $2 \mathrm{~m}$.

\subsection{Infochemical distribution}

\subsubsection{Infochemical dispersion}

In our model study (Lof et al., 2008), we assumed calm weather conditions, that is, no wind (in such weather conditions, the fruit flies are most active). However, even in the absence of an advective wind flow, the air is subject to turbulence. Thus, the infochemicals are dispersed randomly, with a turbulent dispersion coefficient (or eddy diffusivity) that is much larger than the molecular diffusion coefficient. (For small molecules in air, the molecular diffusion coefficient is about $10^{-3} \mathrm{~m}^{2} \mathrm{~min}^{-1}$.) We studied the dispersion of soap bubbles in a closed room, and estimated that $D_{I}=1 \mathrm{~m}^{2} \mathrm{~min}^{-1}$. Note that for the value of the dispersion coefficient, it is not relevant whether the dispersion process takes place in one, two or three dimensional space.

\subsubsection{Infochemical production and emission}

Recently mated females emit $300 \mathrm{ng}$ of Drosophila aggregation pheromone (cis-vaccenyl acetate or cVA) on the substrate in the first 6 hours after mating (Bartelt et al., 1985). This gives an aggregation pheromone deposition on the resource $\left(A_{R}\right)$ of $\theta_{A}=0.83 \mathrm{ng}$ per adult female per minute.

Before the pheromone that is deposited at the resource gets available to the searching fruit flies in the air, it has to evaporate first. This evaporation is a very slow process. In a laboratory experiment, Bartelt et al. (1985) found that $31 \%$ of the original amount of cVA in a dish was still present after 2 days. We used this figure to estimate the evaporation rate $\omega$ of the cVA, assuming a steady linear decay process for the liquid phase (Eq. (11)), which results in a value for $\omega$ of $1.3 \cdot 10^{-4} \mathrm{~min}^{-1}$.

$$
\int_{0}^{2 \text { days }} \omega \exp (-\omega \tau) d \tau=0.31
$$

It is not very obvious how this laboratory evaporation rate should be transferred to field conditions. Probably, evaporation in the field is considerably faster than in the laboratory, firstly because of the higher turbulence (even without wind), and secondly because of heating by sunlight. On the other hand, however, cooling down at night hampers evaporation. As our model aims to simulate field conditions in daytime hours, we use an evaporation rate that is considerably higher than in the laboratory: $\omega=4 \cdot 10^{-4} \mathrm{~min}^{-1}$. 


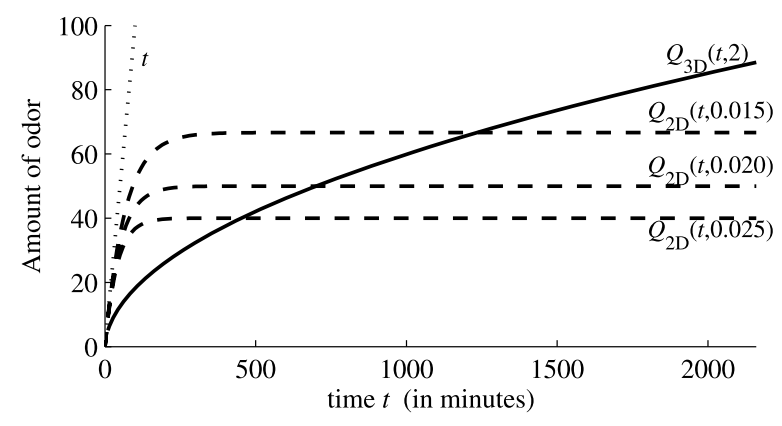

Fig. 1 Odor produced by a unit source as function of the time $t$ (in minutes). Dotted line: total amount produced. Solid line: $Q_{3 \mathrm{D}}(t, 2)$, amount within a $2 \mathrm{~m}$ layer at ground level. Dashed lines: $Q_{2 \mathrm{D}}(t, \mu)$, amount in a $2 \mathrm{D}$ domain with a loss rate $\mu=0.015,0.02$ and 0.025 , respectively.

To quantify the production of food odors we chose ethyl acetate as a characteristic food odor. Yeast infected apples produce this chemical, and D. melanogaster responds to it (Hutner et al., 1937; Echeverría et al., 2003). Echeverría et al. (2003) found that at harvest date, such apples release 4,800 ng ethyl acetate per $\mathrm{kg}$ into the air in 8 hours. Assuming there are approximately 5 apples in $1 \mathrm{~kg}$, this gives an emission of food odors of $\theta_{F}=2$ ng per apple per minute.

\subsubsection{Infochemical loss}

Our fruit fly model is essentially two dimensional in space. This implies that the vertical component is ignored. We envision the physical fruit fly habitat as a $2 \mathrm{~m}$ thick air layer above ground level, and model the number of flies per unit area of the field. Whereas the flies can be considered to be confined to such a layer, the emitted infochemicals obviously are not. Hence, there is a certain loss of infochemicals as they move upward by dispersion, out of reach of the flies. In this section, we discuss this loss rate into the third dimension.

Consider a constant odor source with an emission rate $c(t)(\mathrm{ng} / \mathrm{min})$ starting at $t=0$ at the origin. The amount of odor in a layer with thickness $h$ at time $t$ (in minutes) is

$$
Q_{3 \mathrm{D}}(t, h)=\int_{0}^{t} c(t-\tau) \operatorname{erf}\left(\frac{h}{2 \sqrt{D_{I} \tau}}\right) d \tau .
$$

So, for a constant emission rate $c=1(\mathrm{ng} / \mathrm{min})$ starting at $t=0$,

$$
\begin{aligned}
Q_{3 \mathrm{D}}(t, h) & =\int_{0}^{t} \operatorname{erf}\left(\frac{h}{2 \sqrt{D_{I} \tau}}\right) d \tau \\
& =\left(-\frac{h^{2}}{2 D_{I} t} \operatorname{erfc}\left(\frac{h}{2 \sqrt{D_{I} t}}\right)+\operatorname{erf}\left(\frac{h}{2 \sqrt{D_{I} t}}\right)+\frac{h}{\sqrt{\pi D_{I} t}} \exp \left(-\frac{h^{2}}{4 D_{I} t}\right)\right) t .
\end{aligned}
$$

Figure 1 shows the graph of this function for $D_{I}=1 \mathrm{~m}^{2} / \mathrm{s}$ and $h=2 \mathrm{~m}$, on a time interval of 36 hours. Note that for $c=1$ the total odor released at time $t$ equals $t$.

It is clear that for increasing $t$, there is a quite large and increasing fraction that leaves the layer; initially, however, the loss is quite small. This was to be expected, as the odor 


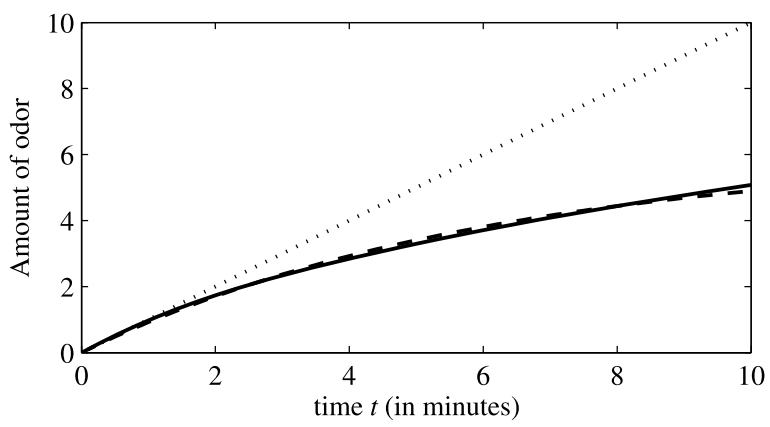

Fig. 2 Odor produced by a unit source as function of the time $t$ (in minutes). Dotted line: total amount produced. Solid line: $Q_{3 \mathrm{D}}(t, 2)$, amount within a $2 \mathrm{~m}$ layer at ground level. Dashed line: $Q_{2 \mathrm{D}}(t, 0.165)$, amount in a $2 \mathrm{D}$ domain with a loss rate $\mu=0.165$.

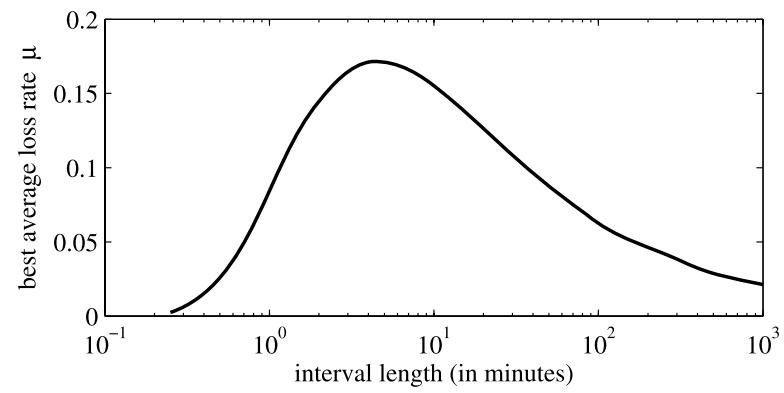

Fig. 3 Average loss rate $\mu$, as a function of the length $t$ of the time interval on which it is used.

is released at the bottom of the layer, and it takes time to get to the boundary at the top of the layer where it can escape. This behavior is essential for the 3D model, and it cannot be captured in a $2 \mathrm{D}$ model.

In an attempt to mimic the $3 \mathrm{D}$ behavior as much as possible, we introduce a loss rate for infochemicals in the $2 \mathrm{D}$ model. With a loss rate $\mu$, the amount of odor from a source with constant emission rate $c=1(\mathrm{ng} / \mathrm{min})$ starting at $t=0$ is

$$
Q_{2 \mathrm{D}}(t, \mu)=\int_{0}^{t} \exp (\mu(\tau-t)) d \tau=\frac{1-\exp (-\mu t)}{\mu} .
$$

Using a constant (nonzero) loss rate causes the available amount in the $2 \mathrm{~m}$ thick layer to be bounded, whereas in the 3D model this amount grows unboundedly as a function of time, see Fig. 1. However, for a small time interval, the 2D loss model can approximate the 3D layer model much more accurately (see Fig. 2).

Given a time interval, we can adapt the loss rate in such way that the time average over that interval for the 2D model with that loss rate and the $3 \mathrm{D}$ model both result in the same amount of odor to disappear from the layer (see Fig. 3).

The resulting function has a maximum approximately at $t=5 \mathrm{~min}$. For larger values, the loss rate must be decreasing to give the proper average value. 
For $t<30 \mathrm{~min}$, the 2D model approximates the 3D model accurately enough. For larger times, the $2 \mathrm{D}$ approximation with the loss rate overestimates the total odor amount in the first half of the interval, and underestimates this amount at later times. Typical average loss rates $\mu(\tau)$ are $0.085,0.165,0.171,0.155,0.109,0.081,0.048$, and 0.025 $\min ^{-1}$ for averaging times $\tau$ of $1,3,5,10,30,60,180$, and 720 min, respectively. In our model, we used $\mu(5)$ for the recently produced odors and $\mu(720)$ for the odors that were already present in the system. Note that all results depend on the layer thickness $h$ and dispersivity $D_{I}$.

\subsection{Fruit fly response to infochemicals}

In modeling the response of fruit flies to infochemicals, the following considerations are taken into account.

1. The strength of smell of the flies depends on the fraction of neuro-receptors that is occupied by infochemical molecules. As the total number of receptors is finite, this implies that there is a saturation effect at high levels of infochemical concentration, which can be represented by a Michaelis-Menten type of response (Postma, 2003).

2. Fruit flies react on food odors and aggregation pheromone in a rather complicated way. Bartelt et al. (1985) showed that for D. melanogaster, the aggregation pheromone is only attractive when food odors are also present; and that the combination of food odors and its aggregation pheromone is about four times more attractive than food odors alone.

Although many sensory response functions are conceivable that are consistent with these considerations, functions that depend only on a linear combination of the concentrations are not among these. A simple relation that is consistent with these findings is given in Eq. (5).

In adopting a sensory response relation, we assume that the fruit flies' sense of direction does not depend on the actual concentration gradients of the infochemicals, but rather on how the fruit flies perceive this gradient. For a relation as Eq. (5), the effect of this assumption is manifest especially at high concentration levels, in the saturation region of the Michaelis-Menten equation. There, the differences in response to concentrations may become so low that the smell loses its direction.

\subsubsection{Attractiveness of infochemicals and attraction ratio}

The attractiveness of infochemicals $(v)$ is a parameter that quantifies the directionality of the movement. Instead of parameterizing $v$ directly, we use $\kappa=v / D_{P}$ as characteristic ratio between the random motion and the active search motion: when $\kappa=0$, movement is random, and the higher the value of $\kappa$, the more the movement is directed toward the odor source. No specific estimate was available for the attractiveness of infochemicals for D. melanogaster. Powell et al. (1998) used $\kappa=10$ for mountain pine beetles. As the population dynamics in our system take place at a smaller spatial scale than the dynamics of the mountain pine beetle, we assumed that $\kappa$ would be smaller, too. We arbitrarily chose $\kappa=5$.

Bartelt et al. (1985) showed that after 3 minutes in a wind tunnel, about four times more adult $D$. melanogaster were attracted by the combination of food odors and the aggregation pheromone than by food odors alone. In a field experiment, Wertheim et al. 


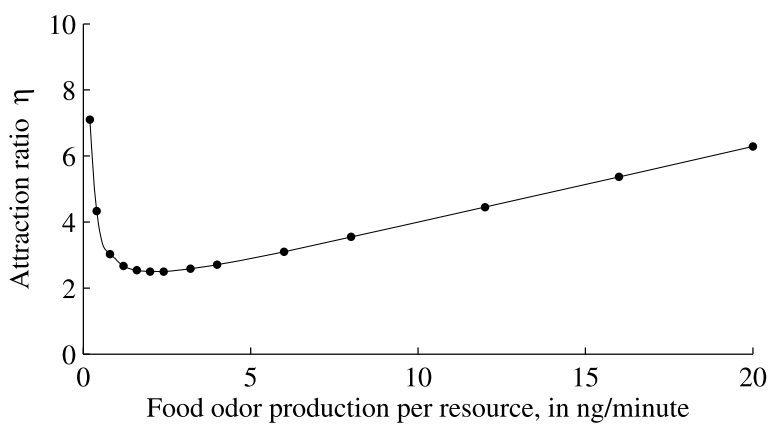

Fig. 4 The combination of pheromones and food odor is 4.5 times as attractive as food odor alone. The corresponding value of $\eta$ depends on the food odor concentration.

(2006) found after 10 minutes a 1:4.5 ratio of fruit fly numbers on substrates with food odor only $(\mathrm{F})$ and food odor combined with aggregation pheromone $(\mathrm{F}+\mathrm{A})$, respectively. These results are very alike. We used Wertheim's 1:4.5 ratio in combination with our chosen $\kappa$ to estimate the attraction ratio $\eta$. Therefore, we set up a simulation on a $2 \mathrm{D}$ domain similarly sized as Wertheim's field experiment, with four F resources containing only apple-yeast mixture, and four $\mathrm{F}+\mathrm{A}$ resources, containing an apple-yeast mixture with $4500 \mathrm{ng}$ synthetic pheromone evaporating at a rate of $\omega=4 \cdot 10^{-4} \mathrm{~min}^{-1}$. Thus, we have a pheromone emission rate of $18 \mathrm{ng} \mathrm{min}^{-1}$. The resources were placed in two rows of four, at regular intervals in alternating order ( $3 \mathrm{~m}$ between rows and $2 \mathrm{~m}$ between resources in a row).

The initial adult population is homogeneously distributed. We ran the simulation for 10 minutes, with time steps of 0.25 minute, for varying values of $\eta$ and for varying values for the production of food odors. Figure 4 shows (for $\kappa=5$ ) the values for $\eta$ corresponding to a 1:4.5 ratio for the average number of fruit flies on the two types of resources for various values of the food odor production. There is a minimum value for $\eta=2.5$ for which the experimental results of Wertheim can be reproduced, at a food odor production of 2.5-3 ng per resource per minute. For all other values of food odor production, the value of $\eta$ must be taken higher.

By coupling $\kappa$ and $\eta$, we kept the attraction ratio constant (at 1:4.5) and, therefore, we could study the sensitivity of the model for our chosen $\kappa$ on its own. In our sensitivity analysis, we simulated for $\kappa=2.5$ with $\eta=5$ and for $\kappa=7.5$ with $\eta=1.67$.

\subsubsection{Half saturation value for aggregation pheromone and food odor}

Wertheim et al. (2006) studied the dose response relation of $D$. melanogaster for cVA. We used their Fig. 1e to calculate the half saturation value of pheromone. This figure depicts the average number of fruit flies found on substrates containing four different doses of cVA $(q=0,0.45,4.5$, and $45 \mu \mathrm{g})$. The number of fruit flies was counted every 10 minutes for 3 hours. We used nonlinear regression to estimate the half saturation value $\left(q_{0}\right)$, using the Michaelis-Menten equation as the model,

$$
P=P_{0}+\frac{b q}{q_{0}+q}
$$


where $P_{0}$ is the number of settled fruit flies settled on substrates without aggregation pheromone, and $b$ is a parameter describing the increase in number of fruit flies due to the presence of aggregation pheromone. We found $q_{0}=3.5 \mu \mathrm{g}$ (with $P_{0}=8.5$ and $b=18.2$ ) as the best solution.

Again, we translate this into concentrations of volatile compounds in the air. Using the previously derived evaporation rate $\omega=4 \cdot 10^{-4} \mathrm{~min}^{-1}$, we approximate the emission rate as $q_{0} \omega$ in the transient phase (up to one day). If the pheromone spreads by a $3 \mathrm{D}$ dispersion process, then its concentration at distance $r$ from the source is

$$
u(r, t)=q_{0} \omega \int_{0}^{t} \frac{\exp \left(-\frac{r^{2}}{4 D \tau}\right)}{(4 \pi D \tau)^{3 / 2}} d \tau .
$$

The length scale of the experiments of Wertheim et al. (2006) was $2 \mathrm{~m}$, and saturation effects were observable after 20 minutes. Therefore, we assume that the $A_{0}$ value as calculated in Eq. (17) is an indication of the half-saturation value for pheromone:

$$
A_{0}=u(2,10)=q_{0} \omega \int_{0}^{10} \frac{\exp \left(-\frac{1}{D \tau}\right)}{(4 \pi D \tau)^{3 / 2}} d \tau=4.2 \cdot 10^{-5} \mu \mathrm{g} \mathrm{m}^{-3}
$$

\section{Parameterization of the model: long term dynamics}

\subsection{Between-generation dynamics: reproduction and survival}

Adult females that have settled on a resource $\left(P_{R}\right)$ deposit on average $\xi$ eggs per minute. The cumulative number of eggs $(L)$ on each resource item after three days (in generation $n$ ) is, therefore,

$$
L(x, y, n)=\int_{0}^{3} \xi P_{R}(x, y, n) d t .
$$

Due to the Allee effect and scramble competition, the probability of successful larvae development depends on the number of larvae and, therefore, on $L$. Hence, the next female population has density $P(x, y, n+1)$,

$$
\begin{aligned}
& P(x, y, n+1)=\varphi L(x, y, n) s_{A}(L) s_{C}(L), \\
& s_{A}(L)=\frac{1}{1+e^{-c_{A}\left(L-L_{A}\right)}}, \quad s_{C}(L)=\frac{1}{1+e^{c_{C}\left(L-L_{C}\right)}},
\end{aligned}
$$

where $s_{A}(L)$ and $s_{C}(L)$ model the reduction of the larvae survival rate because of the Allee effect and the scramble competition, respectively. The sex ratio $\varphi$ is the fraction of females in the larval population.

\subsection{Fecundity}

A study of Boulétreau (1978) showed that D. melanogaster has a mean fecundity of 6 eggs per day in the field. As we use a day length of 12 hours in our simulations, we set $\xi=6$ eggs per $720 \mathrm{~min}=0.0083$ eggs $\min ^{-1}$. 
Table 2 The model parameters involved in the reproduction dynamics

\begin{tabular}{llll}
\hline Name & Description & Value & Units \\
\hline$\xi$ & $\begin{array}{l}\text { Fecundity of the settled population } \\
\text { Sex ratio of the larvae (fraction of } \\
\text { females) }\end{array}$ & 0.0083 & $\min ^{-1}$ \\
& $\begin{array}{l}\text { Number of larvae per apple at which } \\
\text { 50\% survives the Allee effect } \\
L_{A}\end{array}$ & 0.5 & - \\
$L_{C}$ & $\begin{array}{l}\text { Number of larvae per apple at which } \\
50 \% \text { survives competition } \\
c_{A}\end{array}$ & 25 & - \\
Slope sigmoid survival curve \\
modeling the Allee effect \\
$c_{C}$
\end{tabular}

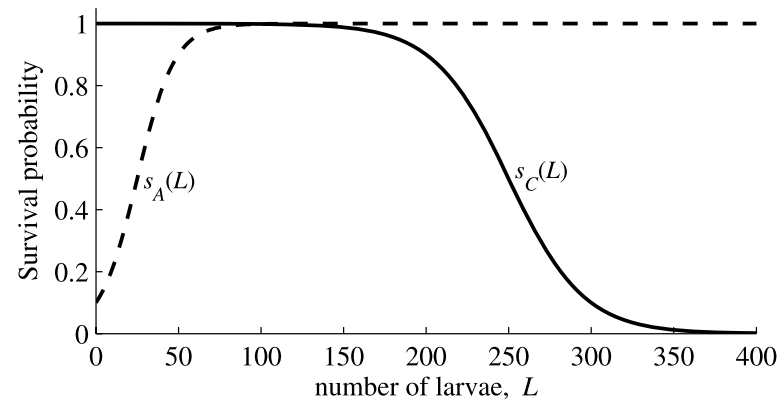

Fig. 5 Larval survival probabilities as functions of the number of larvae on a resource item. The dashed line shows the larval survival related to the Allee effect $\left(s_{A}\right)$, the solid line gives larval survival related to competition $\left(s_{C}\right)$, as given in Eq. (20).

\subsection{Larval survival}

The larval survival probability is modeled with the sigmoid functions $s_{A}(L)$ for the Allee effect and $s_{C}(L)$ for the competition, both of which contain two parameters. In the model, we use yeast-infected apples as the substrate for larval development. One apple can support development of at most 200-300 D. melanogaster larvae (Sang, 1956). We assume that in the interval $(200,300)$ competition reduces larval survival approximately from $90 \%$ to $10 \%$, see Fig. 5. This implies 50\% survival at $L_{C}=250$ larvae and a slope $c_{C}=0.044$.

For the Allee effect, no quantitative data were found in literature. An indication can be found in Rohlfs and Hoffmeister (2003), who observe a high mortality at low larval densities. Therefore, we (arbitrarily) choose $50 \%$ survival at $L_{A}=25$, increasing to $90 \%$ survival at 50 larvae see Fig. 5; this implies a slope $c_{A}=0.088$. 


\section{Sensitivity analysis}

The primary state variables of our model are the three functions of time and position $P_{S}(t, x, y), P_{R}(t, x, y), P_{M}(t, x, y)$, modeling the density of searching, settled and moving fruit flies, respectively. Since the output of the model contains many data, there are numerous ways to measure and weigh the effects of a parameter change in the model. As described in Lof et al. (2008), the model was tested on several time scales: the short term (up to one generation), and the long term (from one to ten generations). It turned out that the long term dynamics displays a chaotic pattern (see Lof et al., 2008, Fig. 7). This behavior is caused by the counteracting effects of the competition and the Allee effect, and it is persistent over a wide range of parameters. Therefore, a sensitivity analysis is not very useful in this situation: the qualitative result, displaying chaotic oscillations, is quite robust, but the quantitative result at any point in time and space may depend critically on the parameter values (and initial conditions).

At the short term, a sensitivity analysis is much more useful. It enables us to investigate how the changes in the parameters of the model affect the fruit fly behavior, especially with respect to its success rate in finding a suitable resource. For this sensitivity analysis, we ran one day simulations as described in Lof et al. (2008), on a symmetric field, starting with 500 flies in the first quadrant, and 100 flies in each of the other three quadrants. For output variables, we compared using the default parameter value and two alternative values,

1. The total number of flies in the first quadrant, $P_{t o t, 1}(t)$, may decrease by dispersion and increase by chemotactically induced aggregation. Therefore, this number acts as an indication of the relative strength of these two opposite effects;

2. The number of settled flies in the first quadrant, $P_{R, 1}(t)$. This number gives an indication of the success in finding resources.

Both $P_{t o t, 1}(t)$ and $P_{R, 1}(t)$ may be used as indicators for aggregation, $P_{t o t, 1}(t)$ on a larger spatial scale than $P_{R, 1}(t)$. We compared $P_{t o t, 1}(t)$ and $P_{R, 1}(t)$ for the different parameter values in two manners: (1) a qualitative comparison of the behavioral dynamics curves of $P_{t o t, 1}(t)$ and $P_{R, 1}(t)$ on the interval $0 \leq t \leq 12 \mathrm{hr}$, and (2) a quantitative comparison of the final values $P_{\text {tot }, 1}(12 \mathrm{hr})$ and $P_{R, 1}(12 \mathrm{hr})$.

\subsection{Fruit fly behavioral parameters}

\subsubsection{Transition rates: $\lambda, \alpha_{1}, \alpha_{2}$}

The settlement rate $\lambda$ (with standard value $0.25 \mathrm{~min}^{-1}$ ) has been varied from 0.125 to $0.375 \mathrm{~min}^{-1}$. This causes the settlement after 5 minutes to vary from $46.5 \%$ to $84.7 \%$, and affects the distribution over the states through the settled population (Table 3). As expected, the settled population increases with an increasing settlement rate.

The model is most sensitive for the patch leaving rate $\alpha_{1}$. Varying $\alpha_{1}$ (with standard value $0.002 \mathrm{~min}^{-1}$ ) between 0.001 and $0.003 \mathrm{~min}^{-1}$ causes a fraction from $0.5 \%$ to $1.5 \%$ of the settled population to leave the resource within 5 minutes. Increasing $\alpha_{1}$ impedes the growth in the number of settled adults considerably over the whole 12 hours interval, and thus causes a strong decrease in the number of settled adults after 12 hours (Table 3). 
Table 3 Sensitivity analysis. The value in the third column is the reference value as used in the simulations. The fourth column contains alternative values for the parameters, all other parameters being on their reference values. The fifth and sixth column give the relative change in percentage of the number of fruit flies after a $12 \mathrm{hr}$ simulation $P_{\text {tot }, 1}(12 \mathrm{hr})$ and $P_{R, 1}(12 \mathrm{hr})$. The seventh and eighth column give a qualitative comparison of the behavioral dynamics of the total and settled population numbers $P_{\text {tot, } 1}(t)$ and $P_{R, 1}(t)$, - means much slower dynamics - means slower dynamics, a blank means approximately equal dynamics, + means faster, ++ much faster dynamics of the population

\begin{tabular}{|c|c|c|c|c|c|c|c|}
\hline Name & Description & Reference value & Alternative values & $P_{\text {tot }, 1}(12)$ & $P_{R, 1}(12)$ & $P_{t o t, 1}(t)$ & $P_{R, 1}(t)$ \\
\hline \multirow[t]{2}{*}{$\kappa \& \eta$} & Attraction ratio & $5.0 \& 2.5$ & $7.5 \& 1.67$ & 2.2 & 3.6 & & + \\
\hline & & & $2.5 \& 5.0$ & -2.2 & -4.0 & & - \\
\hline \multirow[t]{2}{*}{$D_{P}$} & Dispersivity & 0.58 & 0.87 & 0.2 & 1.3 & + & + \\
\hline & of Drosophila & & 0.29 & -3.5 & -7.9 & -- & -- \\
\hline \multirow[t]{2}{*}{$\rho$} & Resource leaving & 1 & 1.5 & -0.4 & -0.6 & & \\
\hline & flight speed & & 0.5 & 0.3 & 0.5 & & \\
\hline \multirow[t]{2}{*}{$\lambda$} & Settlement rate & 0.25 & 0.375 & 1.9 & 3.5 & & \\
\hline & & & 0.125 & -4.8 & -9.5 & & - \\
\hline \multirow[t]{2}{*}{$\alpha_{1}$} & Leaving rate & 0.002 & 0.003 & -5.1 & -10.9 & - & -- \\
\hline & & & 0.001 & 6.9 & 14.2 & + & ++ \\
\hline \multirow[t]{2}{*}{$\alpha_{2}$} & Start-to-search & 0.5 & 0.75 & 0.1 & 0.2 & & \\
\hline & rate & & 0.25 & -0.6 & -1.1 & & \\
\hline \multirow[t]{3}{*}{$D_{I} \& \mu$} & Dispersivity & $1.0 \& 0.14$ & $1.5 \& 0.1$ & -1.5 & -1.3 & & \\
\hline & and loss rate & & $0.5 \& 0.2$ & 5.9 & 5.6 & + & + \\
\hline & of chemicals & & & & & & \\
\hline \multirow[t]{2}{*}{$F_{0}$} & Food odor & 10 & 15 & 3.7 & 4.5 & & \\
\hline & saturation & & 5 & -6.8 & -8.2 & - & - \\
\hline \multirow[t]{2}{*}{$A_{0}$} & Pheromone & 0.04 & 0.06 & 2.9 & 3.0 & & \\
\hline & saturation & & 0.02 & -5.2 & -5.5 & - & - \\
\hline \multirow[t]{2}{*}{$\theta_{F}$} & Food odor & 2 & 3 & -3.8 & -4.6 & - & - \\
\hline & production & & 1 & 6.3 & 7.5 & + & + \\
\hline \multirow[t]{2}{*}{$\theta_{A}$} & Pheromone & 0.83 & 1.215 & -2.8 & -3.0 & - & - \\
\hline & production & & 0.415 & 4.9 & 5.2 & + & + \\
\hline \multirow[t]{2}{*}{$\omega$} & Pheromone & $4.10^{-4}$ & $6.10^{-4}$ & -2.8 & -3.0 & - & - \\
\hline & evaporation & & $2.10^{-4}$ & 4.9 & 5.2 & + & + \\
\hline
\end{tabular}

The rate $\alpha_{2}$ has been varied between 0.25 and $0.75 \mathrm{~min}^{-1}$. Although this has some effect on the rate at which moving individuals start searching at short term (varying between 71.3 and $97.6 \%$ after 5 minutes), it hardly affects the behavioral dynamics or the number of settled adults during or after 12 hours (Table 3 ).

\subsubsection{Fruit fly dispersal: $D_{P}$ and $\rho$}

The dispersal constant $D_{P}$ (with standard value $0.058 \mathrm{~m}^{2} \mathrm{~min}^{-1}$ ) of the fruit fly population has a strong effect on the behavioral dynamics of the settled fruit fly population. For increasing values of $D_{P}$ between 0.029 and 0.087 , the slope of the settled fruit flies graph increases, and hence the numbers of settled fruit flies present after 12 hours increases 
(Table 3). Thus, the farther the fruit flies can disperse the faster fruit flies find a resource and settle.

Varying the velocity $\rho$ of the ring-random dispersal from 0.5 to $1.5 \mathrm{~m} \mathrm{~min}^{-1}$ causes no noticeable effect on the behavioral dynamics (Table 3).

\subsubsection{Fruit fly response: $F_{0}$ and $A_{0}$}

The half-saturation parameters $F_{0}$ and $A_{0}$ concerning fruit fly response toward food odors or aggregation pheromone, respectively, have a moderate effect on both the total and the settled population. Halving the values for $F_{0}$ or $A_{0}$, from 10 to 5 or from 0.04 to 0.02 , respectively, slightly decreases the total number of fruit flies and the number of settled fruit flies after 12 hours (Table 3). Increasing the values for $F_{0}$ or $A_{0}$ by $50 \%$ did not have a great effect. Note that the effect of variation in $F_{0}$ is stronger than that in $A_{0}$. This is due to the fact that fruit flies only react to aggregation pheromone in the presence of food odors. It is reflected in the two terms in the sensory index, one depending on the food odor concentration, the other one depending on the product of food odor and pheromone concentration. Because of its structure, the latter term is equally sensitive to relative changes in food odor concentration and pheromone concentration. In combination with the first term, the sensitivity to relative changes in food odor concentration must be larger than the sensitivity to relative changes in pheromone concentration.

\subsubsection{Attractiveness to infochemicals: $\kappa$ coupled with $\eta$}

As pointed out above, we couple $\kappa$ (the ratio between random movement and odordirected movement) and $\eta$ (the parameter determining the relative attractiveness of pheromone in relation to food odor) in order to keep the 1:4.5 ratio of fruit fly numbers on substrates with food odor only (F) and food odor combined with aggregation pheromone (F+A) as observed by both Bartelt et al. (1985) and Wertheim et al. (2006). Therefore, in the sensitivity analysis, we increase or decrease $\kappa$ by $50 \%$, and adapt $\eta$ in such a way that the above mentioned 1:4.5 ratio is maintained. It is then found that the model was not sensitive to this parameter (Table 3 ).

\subsection{Odor distribution: $D_{I}$ coupled with $\mu$}

Decreasing the dispersal constant $D_{I}$ of the infochemicals to values below $1 \mathrm{~m}^{2} \mathrm{~min}^{-1}$ affects the behavioral dynamics of the total and the settled fruit fly population (Table 3 ). The total population size and the number of settled fruit flies in the first quadrant both increase faster for $D_{I}=0.5$. Decreasing $D_{I}$ thus increases the total number of fruit flies and number of settled fruit flies present after 12 hours. This indicates that fruit flies have less difficulty in finding the odor source when the dispersal of the infochemicals is slow.

As the loss rate $\mu$ is fitted on the dispersal rate $D_{T}$ and the time scale, its sensitivity was not analyzed separately. However, the reduction from 3D to $2 \mathrm{D}$ that is represented by this parameter may have a considerable effect on the model results.

\subsection{Odor production parameters: $\theta_{F}, \theta_{A}$, and $\omega$}

Decreasing the production rate of food odors or aggregation pheromone $\theta_{F}$ or $\theta_{A}$, respectively) had an effect on both the total and the settled population. When the production rate was halved, the total number of fruit flies and the number of settled fruit flies after 12 
hours increased (Table 3). Doubling the value for $\theta_{F}$ or $\theta_{A}$ had no effect. Thus, more fruit flies could find the resources when there were less food odors or aggregation pheromone available in the air. This indicates that in our simulation both infochemicals where available in concentrations that caused saturation of the sensory system of the fruit flies. To understand this phenomenon, one should realize that in our model the fruit flies do not respond to the gradient of the infochemicals concentration itself, but to the gradient of the sensory index of the infochemicals. At full saturation, the sensory index has a constant level, and so its gradient vanishes. Then the fruit flies loose a directional cue, although the infochemical concentration may have a gradient that is quite useful at lower concentration levels. Thus, under conditions of saturation, fruit flies move at random, and have more difficulties in finding the resources.

The standard parameter values that we use are based on the experimental studies of Wertheim et al. (2006). We notice that in the setup in their study, the fruit flies are exposed to large amounts of food odor and pheromone. Therefore, it is not unreasonable that in these experiments the concentration level is close to saturation. From an evolutionary point of view, it seems plausible that the chemotactical abilities of fruit flies are better tuned to finding a resource when resources are scarce than in finding a resource quickly when resources are abundant.

The model was equally sensitive to variation in the evaporation rate of the aggregation pheromone, $\omega$, as to the production rate $\theta_{A}$ (Table 3). Indeed, a clear interdependency of $\omega$ and $\theta_{A}$ can be observed. This is not surprising, as the amount of available pheromone odor that gets available per time unit depends on the product of $\omega$ and $\theta_{A}$.

\section{Conclusion and discussion}

For a spatio-temporal model of Drosophila based on reaction to food odors and aggregation pheromone, realistic parameter values are hard to obtain. In general, any model for the reaction of insects on odor in the air can be divided into three parts:

1. A model for insect behavior where the population members are divided among states depending on their activity.

2. A model for production, evaporation and dispersion of the odor substances.

3. A model for the detection of odors by insects, and their reaction thereupon.

The further investigation and parameterization of these submodels requires input from quite different disciplines.

The first submodel lies in the field of behavioral ecology. As mentioned, the transitions between the three states are known from a biological point of view, because the Drosophila-based food web is already thoroughly studied (e.g., Wertheim, 2001). Quantitative measures of the rate $\lambda$ at which the searching flies $S$ in the close vicinity of a resource item settle, the rate $\alpha_{1}$ at which the settled flies $R$ leave their resource, and the rate $\alpha_{2}$ at which moving flies $M$ start searching again still lack. As these parameters model population processes that have their roots in individual insect behavior, more accurate estimates should be obtainable from insect behavioral experiments, either in artificial setups, or in the field, or both. A study by Wertheim et al. (2002) indicates that fruit flies after an initial choice for a resource mostly stay on that substrate for (at least) 2 hours. Therefore, the choice of 0.02 for the patch leaving rate $\alpha_{1}$ seems reasonable. Still, research on 
the patch residence time of $D$. melanogaster that can be translated to the population level responses is needed. The same applies to oviposition rates of Drosophila.

The second submodel lies in the field of physics, organic chemistry, and micrometeorology. A good measurement of evaporation rates seems to be within reach; also a possible degradation of infochemicals can be studied. Models for plume transport on short and intermediate distances should be investigated further. As we have shown, the reduction of the three-dimensional dispersion of infochemicals to two dimensions may lead to concentration patterns that are qualitatively and quantitatively different. These differences can be remediated only in part with the parameterization. Therefore, it may be interesting to investigate to what extent the results from a full 3D spatial model for insect behavior agree with the results that we find with our essentially 2D model.

The third submodel lies in the field of biophysics and neurology. This submodel may be the most challenging. Although some knowledge exists on how fruit flies react to aggregation pheromone (Wertheim et al., 2002), nothing is known about the spatial dose-response relationship to food odors and aggregation pheromone. In the model for the reaction of fruit flies to infochemicals that we used, two parts may be distinguished: the sensory index function that describes the perception of our virtual fruit flies to infochemicals, and an additional parameter $v$ describing the strength of chemotaxis as compared to random walk movement. At this moment, quantitative data are so scarce that this submodel is clearly overparameterized. We have solved this problem by fixing a combination of the parameters $\kappa$ and $\eta$. It will not be easy to decouple these parameters just by ethological experiments, as this would require a very controlled setup to establish a quantitative relation between fruit fly behavior and measured infochemical concentration gradients.

More fundamentally, one may discuss the type of the sensory index function that we used. For a sensory index function for one infochemical, the use of a Michaels-Menten type of function for saturated response can be made plausible from the limited availability of sensory nerve cells to accommodate infochemical molecules (e.g., Postma, 2003). However, for two interrelating infochemicals, many types of sensory index functions are conceivable, and we have chosen a simple one from them. It may require neurological research on fruit flies to validate this choice.

The sensitivity analysis shows that the model is most sensitive to changes in the fruit fly dispersivity $D_{P}$ and the patch leaving rate $\alpha_{1}$. It should be feasible to obtain better information on these parameters in controlled experiments, either in the field or in the laboratory. A second group of sensitive parameters are the infochemical parameters $\omega$, $\theta_{A}$ and $\theta_{F}$, especially at the lower density levels. This sensitivity might be caused by the sensory response function that we have adopted. However, independent of the sensory response function, this sensitivity highlights the importance of a careful treatment of the infochemical loss.

Insight into the spatial ecology of aggregation should be derived from a multidisciplinary approach that includes the identification of the cues that mediate aggregation, the behavioral responses of individuals to these cues, and the spatial variation in the responses that result from spatial variation in pheromone distribution. With the results of the sensitivity analysis in mind, especially reliable estimates for the parameters $A_{0}, F_{0}$, $D_{I}, D_{P}$, and $\alpha_{1}$ are required. This is exactly what we plan to obtain in future experiments. However, even without these experiments, the sensitivity analysis shows that the overall conclusions in our companion paper (Lof et al., 2008) for the resource density of 5 apples per $\mathrm{m}^{2}$ hold over a wide range of plausible parameter values. The same probably applies for other resource densities. 


\section{Acknowledgements}

The research of Marjolein Lof was sponsored by the Netherlands Organization for Scientific Research, under Grant 635.100.011 of the Computational Life Science program. The authors like to thank Marcel Dicke and two anonymous referees for their valuable remarks.

Open Access This article is distributed under the terms of the Creative Commons Attribution Noncommercial License which permits any noncommercial use, distribution, and reproduction in any medium, provided the original author(s) and source are credited.

\section{References}

Bartelt, R.J., Schaner, A.M., Jackson, L.L., 1985. cis-vaccenyl acetate as an aggregation pheromone in Drosophila melanogaster. J. Chem. Ecol. 11(9), 1747-1756.

Boulétreau, J., 1978. Ovarian activity and reproductive potential in a natural population of Drosophila melanogaster. Oecologia 35(3), 319-342.

Echeverría, G., Fuentes, M.T., Graell, J., López, M.L., 2003. Relationships between volatile production, fruit quality and sensory evaluation of Fuji apples stored in different atmospheres by means of multivariate analysis. J. Sci. Food Agr. 84(1), 5-20.

Etienne, R., Wertheim, B., Hemerik, L., Schneider, P., Powell, J.A., 2002. The interaction between dispersal, the Allee effect and scramble competition affects population dynamics. Ecol. Model. 148(2), $153-168$.

Hutner, S.H., Kaplan, H.M., Enzmann, E.V., 1937. Chemicals attracting Drosophila. Am. Nat. 71(6), 575581.

Lof, M.E., Etienne, R., de Gee, M., Hemerik, L., Powell, J., 2008. The effect of chemical information on the spatial distribution of fruit flies: I Model results. Bull. Math. Biol. doi:10.1007/s11538-008-9327-0.

Parrish, J.K., Edelstein-Keshet, L., 1999. Complexity, pattern, and evolutionary trade-offs in animal aggregation. Science 284(\#5411), 99-101.

Postma, M., 2003. Spatial and temporal aspects in biological signal transduction: diffusion, reaction and geometry in Drosophila phototransduction and Dictyostelium chemotaxis. $\mathrm{PhD}$ thesis, University of Groningen, Groningen, The Netherlands.

Powell, J.A., McMillan, T., White, P., 1998. Connecting a chemotactic model for mass attack to a rapid integro-difference emulation strategy. SIAM J. Appl. Math. 59(2), 547-572.

Rohlfs, M., Hoffmeister, T.S., 2003. An evolutionary explanation of the aggregation model of species coexistence. Proc. R. Soc. Lond. B 270(Suppl.), S33-S35.

Sang, J.H., 1956. The quantitative nutritional requirements of Drosophila melanogaster. J. Exp. Biol. 33(1), 45-72.

Timofeeff-Ressovsky, N.W., Timofeeff-Ressovsky, E.A., 1941. Populationgenetische Versuche an Drosophila II. Aktionsbereiche von Drosophila funebris und Drosophila melanogaster. Z. Induct. Abstamm. Ver. 79, 35-43.

Wertheim, B., 2001. Ecology of Drosophila aggregation pheromone: a multitrophic approach. PhD thesis, Wageningen University, Wageningen, The Netherlands.

Wertheim, B., Dicke, M., Vet, L.E.M., 2002. Behavioral plasticity in support of a benefit for aggregation pheromone use in Drosophila melanogaster. Entomol. Exp. Appl. 103(1), 61-71.

Wertheim, B., van Baalen, E.J.A., Dicke, M., Vet, L.E.M., 2005. Pheromone-mediated aggregation in nonsocial arthropods: an evolutionary ecological perspective. Annu. Rev. Entomol. 50, 321-346.

Wertheim, B., Allemand, R., Vet, L.E.M., Dicke, M., 2006. Effects of aggregation pheromone on individual behaviour and food web interactions: a field study on Drosophila. Ecol. Entomol. 31(3), 216-226. 